\title{
Goal synchronization of bimanual skills depends on proprioception
}

\author{
Oleg V. Kazennikov ${ }^{a}$, Mario Wiesendanger ${ }^{b, *}$ \\ a Institute for Information Transmission Problems, Neurobiology of Motor Control Russian Academy of Sciences, \\ Bolshoy Karetny 19, 101447 Moscow, Russia \\ ${ }^{\mathrm{b}}$ Laboratory of Motor Systems, Department of Neurology, University of Berne, Inselspital, \\ CH-3010 Berne, Switzerland \\ Received 1 April 2005; received in revised form 29 May 2005; accepted 17 June 2005
}

\begin{abstract}
The present experiments in Human subjects were designed to test whether proprioceptive feedback plays a role in optimising bimanual synchronization in a goal-oriented familiar task. Goal-synchronization is a typical feature of bimanual everyday skills. The purpose of the study was to disturb proprioceptive signalling by means of vibrating the leading left limb while subjects performed a bimanual task on a drawer manipulandum. Blindfolded subjects reached for and opened the drawer with the left hand while the right hand was reaching for grasping an object as the drawer was fully opened. Discrete events of the task were used to measure movement onset times of pulling and grasping hands and of goal arrival times. A spatial-temporal goal invariance was still present despite asymmetrical limb assignments and subjects were blindfolded. In contrast, when vibration $(80 \mathrm{~Hz})$ was applied to the forearm flexors of the leading pulling limb, we found that the interval between the hands at goal reaching was significantly prolonged. This suggests that synchronization is not predetermined entirely by feedforward commands and that proprioceptive feedback is necessary for updating an internal forward model and perhaps also for lower-level corrections in order to ensure covariant limb movements for optimal goal-synchronization.

(c) 2005 Elsevier Ireland Ltd. All rights reserved.
\end{abstract}

Keywords: Bimanual synchronization; Vibration; Proprioception; Motor equivalence

Buttoning a shirt, lacing shoes, or eating with fork and knife are examples of the many bimanual skills of everyday life. Such familiar and well-practiced tasks are performed flawlessly and semi-automatically without much attention. And yet these skills, learned in early childhood, require a precise, spatially and temporally coordinated action of both hands. Patients suffering from movement disorders, such as apraxia, habits of everyday life may become a great problem. Permanent loss of cutaneous and deep sensibility in the upper limbs has disastrous consequences on skilled performances, particularly in the dark (e.g. [2]). Coordination of familiar skills depends not only on memorized central representations and visual guidance but also on sensory feedback from the periphery. In deafferented patients, lack of feedback for updating

* Corresponding author. Present address: Department of Physiology, University of Fribourg, Rue du Musée 5, CH-1700 Fribourg, Switzerland. Tel.: + 4126402 4528; fax: +41 263009734 .

E-mail address: neuro@freesurf.ch (M. Wiesendanger). internal predictive models prevents subjects to deal proactively with interaction torques $[8,10]$.

Bimanual coordination has been studied mostly in rhythmic movements, such as finger tapping and circling, that are not object-related. In the past, we studied bimanual coordination by means of a drawer-task, consistent with familiar everyday manipulations. It allowed us to assess quantitatively the temporal structure of the goal-directed bimanual synergy and proved to be an excellent model for studying bimanual coordination. The task consisted of reach-pull movements of the leading hand and reach-grasp movements of the partner hand. The action was conceived as a single goal-directed synergy. We observed that in both, monkeys [7] and human subjects $[6,9]$, the hands were well synchronized at the goal in spite of their asymmetric roles. This occurred in the face of considerable variability in the timing of the single limb components. We could demonstrate that synchronization was based on strong temporal co-variation of the two limbs. It was also the case, when vision was excluded. Moreover, subjects 
performed the task as a unified synergy, rather than moving sequentially. Synchronization intervals at the goal were similar or even shorter than at the start of the synergy, suggesting that 'on-line' corrections occur while the bimanual synergy is unfolding [6]. Relative invariant goal achievement with variable limb trajectories is the hallmark of volitional goaldirected actions [1]. To our knowledge, bimanual everyday tasks in deafferented patients have not been formally tested.

Full opening of the drawer with the pulling hand is conditional for grasping the object with the other hand. The resulting leading status of the pulling hand over the picking hand (50-100 ms) suggests that any physiological feedback, generated by proprioceptive signals of the pulling limb may contribute in coordinating the two limbs. We hypothesized that a vibration-evoked barrage of excited proprioceptors and cutaneous receptors in the leading limb looses its physiological function of coordinating the picking hand with the pulling hand. As a consequence, one would expect a deterioration of goal-synchronization.

Nine healthy subjects participated in the bimanual drawer task (one female, eight males, aged between 25 and 68 years). All subjects were right-handers according to the Edinburgh handedness inventory. In order to exclude confounding effects of vision, subjects were blindfolded during all experimental series.

The experimental set-up and the drawer manipulandum for the bimanual pull-and-grasp task were the same as used in previous experiments [6,9]. Fig. 1 illustrates the task and its temporal course with time markers indicating goal achievement of the left and right hand. In brief, subjects sat in front of a horizontal platform, elbows flexed at about right angles. Both index fingers were placed on elevated disks defining the start positions near the midline, $30 \mathrm{~cm}$ in front of the chest. After the go-signal, subjects had to reach out for grasping the flat handle of the drawer, and to pull it open at preferred speed (pulling distance $=7 \mathrm{~cm}$ ). The picking arm also reached out to the opened drawer, picked up and reinserted a small peg with the precision grip. All subjects chose to use the left hand for pulling (pulling hand) and the right hand for picking up the peg from the opened drawer (picking hand). Lifting the fingers from the touch-sensitive start positions generated electronic pulses as event markers of movement onset of the left and the right hand. Goal reaching of the leading pulling hand was defined by the moment of complete drawer opening, measured by a position transducer of the drawer. Goal reaching of the picking hand was defined by the event marker when the index finger entered the drawer with the precision grip, thus interrupting a light beam (Fig. 1B). From the arrival times of the two hands, we calculated the interlimb interval at goal reaching, i.e. goal synchronization. A bias load was imposed on the drawer to oblige subjects to keep the drawer open until the peg was reinserted. Proprioceptive and cutaneous afferents of the pulling arm were activated by a vibrator at $80 \mathrm{~Hz}$ (Dynatronic VB100, available frequencies: $25-125 \mathrm{~Hz}$; excursions $0.5 \mathrm{~mm}$ ). The cylinder-like assembly (38 mm diameter, $75 \mathrm{~mm}$ long, $125 \mathrm{~g}$ weight) was fastened,

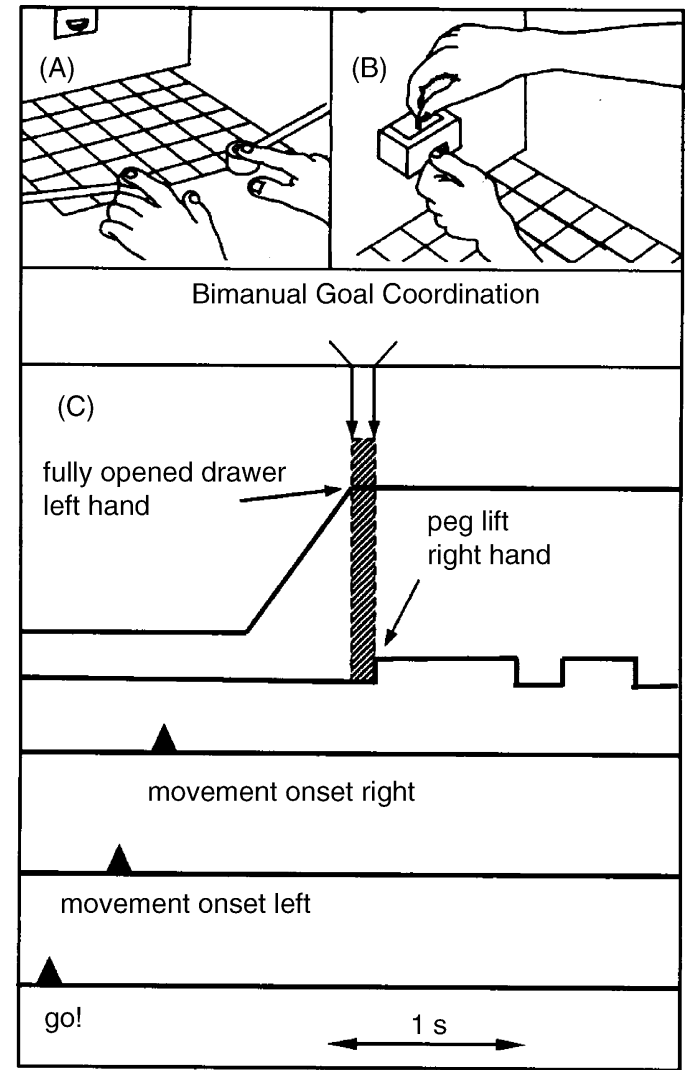

Fig. 1. Bimanual drawer task with start position (A) and goal reaching (B). The left hand first reaches out to the drawer handle and pulls the drawer to The left hand fist reaches out to the drawer hande and pulls the drawer to its mechanical stop while the right hand reaches to the drawer as it opens and picks up a small peg with the precision grip from the drawer recess. (C) Schematic diagram of the event sequence as electronically recorded and measured to assess the temporal structure. Example of one trial with the indicated events (black triangles, from bottom up) and a schematic drawing of the drawer displacement by left hand (from closed to fully opened position): (1) go signal; (2) movement onset left hand; (3) movement onset right hand; (4) peg lift with right hand rectangular elevation of event-marker indicating the duration of the peg-lift (followed by a shorter peg re-insertion). (5) Drawer displacement: transition from closed to fully opened position (left hand). Interlimb synchronization at the goal, the main variable of interest, was determined by the time difference between the event drawer fully open

with the one-sided flat surface over the forearm flexors near the origin of the tendons, or over the thenar in alternating series. These two sites were chosen because both would be involved in the pull and grasp action. After minimal training, subjects were perfectly capable to do the task without vision. The blindfolded subjects performed three runs of 20 trials each, in a balanced sequence among subjects: a series without vibration (control), a series with vibration of long wrist- and finger flexors, and a series with vibration of the thenar. Vibration was applied during the entire single trial (onset was manually triggered just before the 'go-signal' and stopped immediately after the hands were back at the start positions). The procedures have been approved by the local ethical committee and subjects gave informed consent to participate in the study. 


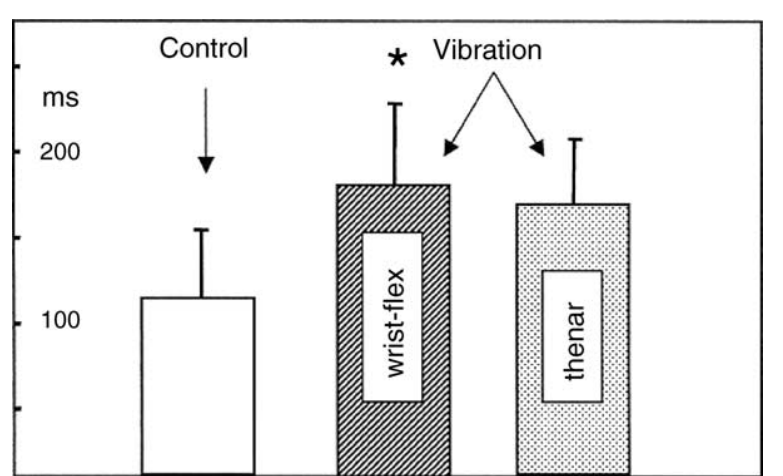

Fig. 2. Mean scores of synchronization at the goal obtained under the 3 conditions. Significant desynchronization occurred when the tendons of the forelimb finger/wrist flexors (wrist-flex) were vibrated $\left(\boldsymbol{\star}_{p}<0.05\right.$ ); the change with vibration of the thenar muscles (thenar) did not reach significance.

Data acquisition: The event signals, together with the drawer position, were fed into separate channels of a laboratory computer using an interface (SC/ZOOM, Department of Physiology, Umeå, Sweden). Statistical analysis consisted of a one-way ANOVA with repeated measures on the conditions of no-vibration, proximal vibration (wrist flexors), and distal vibration (thenar), with mean value of goal-synchronization as the dependent variable. The significance of differences was set at $p<0.05$.

All subjects, blindfolded throughout the three experimental runs, had no difficulty to execute the series. But some subjects felt that vibration interfered somewhat with their movements. Fig. 2 shows that vibration significantly affected bimanual goal synchronization $\left(F_{2,16}=3.932, p<0.05\right)$. Post hoc analysis (Tukey) further revealed that desynchronization was significant only for vibrating forearm flexors $(p<0.05)$. Thenar vibration also tended to desynchronize the two hands at the goal, but failed to reach significance, even with the least conservative post hoc tests (Fisher LSD, Bonferroni, Newman-Keuls, Duncan, $p=0.063$ ).

The results are in line with the hypothesis that proprioceptive signals contribute to goal-synchronization in the bimanual drawer task. The established vibration-induced desynchronization, as compared to the control condition without vibration, was limited to goal achievement. Although vibration was applied from the start, it did not interfere significantly with interlimb synchronization at movement initiation. This indicates that, at the goal, the impaired synchronization was not caused by a late start; rather it was due to the (vibration-induced) disturbed feedback from the pulling limb during its move to the goal. Furthermore, it is suggested that the duration of the synergy from start to goal (about 1.2 s) was long enough to allow for interlimb temporal adjustments in an 'on-line' mode.

In view of the highly disturbed coordination in patients with deafferented limbs, e.g. [4], it was becoming clear that sensory feedback plays a role also in familiar (so-called 'overtrained') skills, such as the dynamic adjustments in object manipulations with the precision grip (for recent overview, e.g. [12]). In chronically deafferented patients, however, the situation may be more complex since secondary degenerations and other plastic changes are likely to occur in the central nervous system. A case report of a patient who suffered from a 'dense hemianesthesia of the left arm', caused by a cerebrovascular parietal cortical lesion, suggested that the patient's bimanual actions of everyday life were abnormal, with gross directional and tempora irregularities in natural bimanual actions. Interestingly, the affected arm was unimpaired in unimanual tasks [5]. In bimanual rhythmic circling movements, vibratory stimuli increased the normal delay between the leading dominant hand and the non-dominant hand [11].

There are three issues that deserve some further comments: (1) The lead of one limb over the other in skilled and object-related bimanual actions. It is clear from the present and from our previous bimanual drawer experiments that the left limb plays a leading role. First, in all our bimanual experiments the great majority of subjects spontaneously chose the left hand to pull open the drawer. Second, the left reach movement typically preceded the right reach and picking movement. The right limb, however, appeared to be more flexible, adjusting its timing according to the progress of the left limb. This has previously been observed when the thumb and the index finger, used as a pinch on the drawer handle, were locally anaesthetized [6,9]; this resulted in a severe delay of the left pulling action. Facing this delay, the right picking hand adjusted the timing by prominent detour trajectories in order to match its arrival with that of the left hand for bimanual goal synchronization. Subjects were not aware of this adjustment. This bimanual matching at the goal was not due to visual guidance since synchronization was usually even better without vision. In the present experiments, however, the vibration effects were not matched with similar manoeuvres as in the previous experiments, suggesting that the right hand had no knowledge about the progress of the leading left limb. On these grounds, we like to speculate that in similar bimanual skills, it is the leading left limb that imposes the timing of goal achievement (temporal goal invariance), whereas the right limb appears to exce in temporal adaptability. (2) About the nature of intermanual coordination. In the light of the above goal invariance, questions arise about mechanisms of interlimb communication: is there a low-level transfer of receptor activity from the leading limb to the opposite limb, a reflex-like transmission? or is the temporal adjustment more complex, involving supraspinal, perhaps even cortical levels as suggested for a forward internal model? (3) The issue of involved receptors: vibration is spreading widely in the tissue and is known to activate not only muscle spindles, but also several types of dynamic skin receptors. Functionally, all of these dynamic receptors, including cutaneous receptors, may contribute to reflex-like adjustments, as was well documented for the dynamic and reflex-like regulation of grasping actions $[3,12]$. 
In conclusion, there is converging evidence that the timing of bimanual manipulation is accompanied by feedback-based temporal adjustments, possibly also by updating a feedforward model. To our knowledge, the present results have shown for the first time that, in a natural and goal-directed bimanual synergy, introduction of perturbing vibration to one limb tends to impair synchronization of the two limbs at the goal. Obviously more has to be learned about the mechanisms of bimanual coordination, especially in view of the variable limb components that lead to a unitary goal invariance. Cooperation of the two hands make up most of our skilful manipulations.

\section{Acknowledgement}

The research was supported by the Swiss National Science Foundation through its NFP-38 program (grant no. 4038-044053 to M.W.)

\section{References}

[1] J.H. Abbs, K.J. Cole, Neural mechanisms of motor equivalence and goal achievement, in: S.P. Wise (Ed.), Higher Brain Functions, Wiley, New York, 1987, pp. 15-43.

[2] C. Bard, J. Paillard, Y. Lajoie, M. Fleury, N. Teasdale, R. Forget, Y. Lamarre, Role of afferent information in the timing of motor commands: a comparative study with a deafferented patient, Neuropsychologia 30 (1992) 201-206.
[3] B.B. Edin, G. Westling, R.S. Johansson, Independent control of human finger-tip forces at individual digits during precision lifting, J. Physiol. 450 (1992) 547-564.

[4] C. Ghez, J. Gordon, M.F. Ghilardi, R. Sainburg, Contributions of vision and proprioception to accuracy in limb movements, in: M.S. Gazzaniga (Ed.), The Cognitive Neurosciences, MIT Press, Cambridge, MA, 1995, pp. 549-564

[5] G.M. Jackson, S.R. Jackson, M. Husain, M. Harvey, T. Kramer L. Dow, The coordination of bimanual prehension movements in a centrally deafferented patient, Brain 123 (2000) 380-

[6] O. Kazennikov, S. Perrig, M. Wiesendanger, Kinematics of a coordinated goal-directed bimanual task, Behav. Brain Res. 134 (2002) 83-91.

[7] O. Kazennikov, U. Wicki, M. Corboz, B. Hyland, A. Palmeri, E.M. Rouiller, M. Wiesendanger, Temporal structure of a bimanual goaldirected movement sequence in monkeys, Eur. J. Neurosci. 6 (1994) 203-210.

[8] D.A. Nowak, S. Glasauer, J. Hermsdorfer, How predictive is grip force control in the complete absence of somatosensory feedback? Brain 127 (2004) 182-192.

[9] S. Perrig, O. Kazennikov, M. Wiesendanger, Time structure of a goal-directed bimanual skill and its dependence on task constraints, Behav. Brain Res. 103 (1999) 95-104.

[10] R.L. Sainburg, M.F. Ghilardi, H. Poizner, C. Ghez, Control of limb dynamics in normal subjects and patients without proprioception, J. Neurophysiol. 73 (1995) 820-835.

[11] M. Steyvers, S.M. Verschueren, O. Levin, M. Ouamer, S.P. Swinnen, Proprioceptive control of cyclical bimanual forearm movements across different movement frequencies as revealed by means of tendon vibration, Exp. Brain Res. 140 (2001) 326334.

[12] A.G. Witney, A. Wing, J.L. Thonnard, A.M. Smith, The cutaneous contribution to adaptive precision grip, Trends Neurosci. 27 (2004) $637-643$. 